\title{
Plants Disease Segmentation using Image Processing
}

\author{
Rabia Masood \\ Shaheed Zulfikar Ali Bhutto Institute of Science and Technology, Islamabad, Pakistan. \\ E-mail: rmasood@numl.edu.pk \\ S.A. Khan, M.N.A. Khan \\ Shaheed Zulfikar Ali Bhutto Institute of Science and Technology, Islamabad, Pakistan. \\ E-mail:mnak2010@gmail.com
}

\begin{abstract}
The image segmentation performs a significant role in the field of image processing because of its wide range of applications in the agricultural fields to identify plants diseases by classifying the different diseases. Classification is a technique to classify the plants diseases on different morphological characteristics. Different classifiers are used to classify such as SVM (Support Vector Machine), K- nearest neighbor classifiers, Artificial Neural Networks, Fuzzy Logic, etc. This paper presents different image processing techniques used for the early detection of different Plants diseases by different authors with different techniques. The main focus of our work is on the critical analysis of different plants disease segmentation techniques. The strengths and limitations of different techniques are discussed in the comparative evaluation of current classification techniques. This study also presents several areas of future research in the domain of plants disease segmentation. Our focus is to analyze the best classification techniques and then fuse certain best techniques to overcome the flaws of different techniques, in the future.
\end{abstract}

Index Terms-Plant disease segmentation, morphological characteristics, features extraction, neural Networks, Fuzzy Logic.

\section{INTRODUCTION}

There are so many things that can cause different diseases to the plants, which damaged big crop fields and ultimately the economy of the country is affected. If plants diseases are detected on the early basis and prevented accordingly, then big losses can be avoided. So to strengthen the agricultural fields and the economy of the country, rapid and accurate detection of plants diseases is needed.

There are lots of techniques in use in order to detect the different diseases of plants in its early stages. Among those techniques, the image processing techniques are becoming very efficient and reliable day by day. To save time, efforts, labors and use of heavy pesticides there is a need of fast and accurate diseases detection of plants. For better quality and quantity of crops in agricultural fields different techniques are proposed by different researchers with the help of digital image processing for rapid and accurate plants disease identification. This paper is survey of different imaging techniques used for the early detection of plant's diseases by different researchers and the limitations of these techniques are also discussed in the end of paper.

\section{LITERATURE REVIEW}

In [1], the authors proposed an automatic and good solution to detect and classify the plants diseases automatically in an accurate and speedy manner. In this technique the pixels are classified on the basis of a set of features in to $\mathrm{K}$ number of classes. A leaf image is divided in to 4 clusters. When the leaf is affected by more than one disease then there will be one or more clusters that will have the disease. $\mathrm{K}$ means algorithm performs the segmentation process in this way. Then Extraction of sets of features by color co occurrence method to analyze texture of the affected leaves was done. Detection and then classification of diseases, s types using ANNs was done. In this method Artificial Neural Networks are used for the purpose of leaves diseases automatically. It is seen that $94 \%$ accuracy is achieved over $89.5 \%$. In addition $19 \%$ more speedy computation time is achieved which was a big achievement.

In [2], the author proposed a technique in which expanse and grading time is reduced as compared to manual system. The proposed methods follow three steps: 1. Pre processing of image. 2. Segmentation of image. 3. Statistical analysis. First image is acquired and then color image vector median filter is used to preprocess the image of crop leaves. The processed image of crop disease is selected on the basis of affected and normal area color difference. Then normal and affected areas are segmented by statistical pattern recognition. Then ratio percentage of the normal and affected area is calculated. Finally the degree of severity of crop disease is found out. During preprocessing of image color information method is used instead of traditional image enhancement method. With the use of improved vector median filter better edges are achieved. To segment the image the statistical pattern recognition classifier was used. By the use of this 
classifier the crop disease images of corn, grape and cucumber were separated from the back ground. Good segmentation was achieved because the area can be divided by the normal area. To reduce the noise from that identification open and close mathematical operation were performed. The accuracy of grading was improved while time and cost were reduced. Hardware requirement was very low.

In [3], the author chosen certain types of crops including rose, cucumber, capsicum, tomato, etc and cultivated them in a green house. Different types of pest including white flies, aphids, thrips, etc affected these crops in different ways. In this paper the authors focused on sooner detection of plants diseases and compared the proposed method with some of the currently used methods. The proposed method has certain steps: 1 . Acquisition of image. Here the images are captured by the camera and the camera is linked with the system. 2 . Pre processing of image. Image is enhanced in this step. Image pre processing is again done by the following three steps: (i). RGB Conversion in to Gray scale. In RGB each pixel is made by three component i.e, red, green and blue. So more space and time is required for RGB. That's why RGB image is converted to Grayscale image. (ii) Resizing of image. Images are resized according to the need. For resizing of images nearest neighbor interpolation, bilinear and bi cubic methods are used. No other pixels except that pixels which falls within are taken in nearest neighbor. In Bi linear the resultant pixel is the weighted average of pixels. (iii). Image filtration. This is the process of cleaning up of an image and highlighting some information. Low pass, High pass and Median filters are used for this. Smoothening filters are used to smooth the edges. The simple Smoothening filter is the average filter having $3 / 3$ matrix. 3. Extracting features. Co-special mask. The classification process is done with the help of gray co occurrence and region properties. SVM is then used for categorization. In Conclusion it is said that the proposed system is reliable for sooner pest's detection.

In [4], the author view is that the plant diseases may be of three type's i.e, viral, fungal and bacterial. The author suggested the proposed method for fast detection and classification of plant's diseases. According to the given method first the images are taken by the digital camera. Image processing techniques are applied to analyze and collect some important features. We have certain steps: (i) The RGB images of all the leaves samples are collected. (ii) RGB image is converted in to HSI (Hue Saturation Intensity) format. (iii) Masking of green pixels is done. For masking of green pixels first of all green pixels are identified. Then mostly green pixels are masked based upon a specific thresh hold values. These values are computed for these pixels. If green component of pixel is less than the already calculated thresh hold value, then the red, green, blue pixels are assigned as zero. Because the green colored area means that these are the healthy parts of the leaves. (iv) Removing the masked green pixels. For better disease classification in minimum time the pixels with zeros red, blue and green were completely removed. (v) Getting useful segments. For this segmentation process the affected part of the leaf only is extracted. Process of segmentation of affected areas is done in equal size portions. Not all segments have important information so only those segments that have useful data are picked only. For analysis only those segments are considered that have more than $50 \%$ information. (vi) Color co-occurrence method is used to compute the features. For color co occurrence, the SGDM (Spatial Gray level Dependence Matrices) is used. In SGDM statistical sampling is done. Some gray levels are related with some other gray levels. (vii) Evaluation of texture statistics is done in the end. Energy, Contrast, Local homogeneity, Cluster shade and Prominence are some important features which are calculated for $\mathrm{H}$ component of the image. All these texture features are divided in different types.

In [5], the authors used K-Means clustering algorithm and correspondence filter to identify the pests. First they mentioned the different current researches and then they mentioned that the combined effect of both the methods (K-Means clustering algorithm and correspondence filter) for automatic identification of pests. The authors have tested 10 different pests. The proposed method for pest's detection is divided in to two steps: (1) Detection and (2) Recognition. In the First phase of algorithm images are captured first and then these captured pictures are loaded as the filter construction images. Now for RGB pests images color transformation structure was formed. Color space transformation was applied then. For pests segmentation k-means clustering was used. The process of pest's segmentation was started by identifying green colored pixels depending upon different threshold values. The Red, Green and Blue color components of the pixels which are less than the fixed thresh hold values are assigned zero value to ignore such values. Because such type of pixels are not important for identification of pests. All the pixels that were on the edges and colored pixels that have assigned zero values were completely removed. The same process was repeated for every pest using in this case. In the Second phase of algorithm angle multiplexing was done for those pests that had detected with the help of K-means clustering algorithm in to the corresponding filter at 5 degree increments. The resultant images were normalized at 3D plot from the correspondence filter and were displayed. The location where pest's images were correlated in a good way resulted a maximum correlated height almost equal to 1.0 and above and was shown by the peak in the matrix. The results achieved have proved that the homogeneous objects were present which further proved that the given algorithm was an effective pest's identification and recognition algorithm. The reliability of the suggested algorithm was proved as the best because the suggested filter could respond to in plane distortion of the pests from 00 to 3600. The correspondence filter was used for distortion in variance ability for accuracy of detecting and recognizing. The sub data base was composed of 10 training images rotated by 5 degree increment in the plane. Good values of tuning parameters alpha, beta and 
gamma were selected for the purpose that the correspondence filter could do recognition job in a good manner.

In [6], the authors suggested the statistical procedures to detect first and then classify the fungal diseases. The effects of fungal diseases are observed on different fruits like pomegranates, grapes and mangoes. Infected fruit images by fungal diseases are first taken and then classified depending upon the extreme position of mentioned diseases like, normal, infected, moderate infected and severe infected. Different statistical features like block wise, Gray Level Co occurrence Matrix, Gray Level Run length Matrix are extracted from these images. Euclidean is used to classify these images, as normal, partially infected, moderately infected and severely infected. GLCM and GLRM have 91.37 and 86.715 percentages for classification accuracies but because of block wise features the average classification accuracy has been improved to 94.085 percent. The authors proposed a method for the given task in which images are captured first, preprocessing, and features extraction and finally classification was done. Image set was consisted of 929 image samples of fruits (grapes, pomegranates and mangoes) infected by fungal diseases. Different fungal disease symptoms on different fruit types were there. Different portions of the plant like stem, leaf and fruit which could be infected from different fungal diseases were considered. Different fungal infected images were then classified in to 300 normal, 215 severely infected, 168 moderately infected and 246 partially infected. For preprocessing one infected image was acquired by the analog camera. Deleting artifacts, shade correction, formatting, edge detection and binarization are the steps of preprocessing. Formatting is related with the storage representation and setting the attributes of the image. The formatted was used as an input to the binarization and edge detection. The preprocessing was done in two stages. In the first stage, binarization and noise deletion was done by the median filter. The filtered image was resized to a constant resolution of $30 \times 30$ sizes. The image was thinned and finally bounding box was produced. In the second stage edge detection was done by the canny edge detector. The process of getting the statistical values from the image by some calculation is the Feature extraction. Statistical based feature extraction methodology was used in this paper. Texture is a useful feature for many natural surfaces and naturally occurring patterns. The intensities are produced by a 2 - dimensional random field. The method was dependant on spatial frequencies and also yields characterization of textures such as grainy, smooth and coarse. GLCM and GLRM which are second order texture features were used for analysis of texture. These methods were compared then with the first order statistical feature like block wise. This processed image was given as an input to block wise, GLCM and GLRM feature extraction methods. In block wise feature extraction the features were extracted from each row and column from block of $5 \times 5$ sizes. The GLCM was based on some repeated occurrences of some gray levels in the texture. The feature extraction was done in two steps, one is the development of the run length matrix and another is the calculation of the features depends on the run length matrix. For classification a nearest neighbor classifier was used. It was a very simple classifier. In this classification, classification of pattern was done depending upon the class label of the closest training patterns in the feature space. Every time a test pattern was to be classified by comparing by comparing with all the training patterns so that the closest training pattern could be found. Proximity measures were used to find similarity or dissimilarity patterns. Euclidean distance was used because it is easy for human interpretation, translation invariant and rotation. Statistical features were extracted from fungal infected samples of fruit image in this training stage. These extracted features were stored in the database. For each class the classifier was tested on the test images. The test image was classified to be related to a specific class to which its Euclidean distance was minimum in all the calculate distances. For the results, Matlab 7 was used. The samples of images were divided in to two portions one is for the training and another is for the testing. For testing and training the image samples were picked at random. Experiments were performed ten times for each training and testing and classification was calculated each time.

In [7], the authors suggested an automatic procedure for detecting affected leaves and severity estimation of the coffee leaves by preventing defoliation. LUT depending gamma corrections are used to enhance the contrast of the actual image and then the background was removed. In $\mathrm{V}$ channel of YUV, fuzzy c-means segmentation in space color was used to get the clustered output image. This was done by maximizing all the damage detection of leaves and then extreme effect of the leaves was measured in the form of ratio between the normal and the identified infected leaves. This was to overcome different challenges faced during capturing and detecting infected leaves. As the authors mentioned different researches which were not sufficient to identify the affected leaves more accurately. The authors suggested a procedure that has an algorithm consisted of the three phase: an automated segmentation of Contrast enhancement, an image of the relative brightness and darkness of the objects are adjusted to enhance the visibility. The suggested procedure could be handled with any image without taking care of its position of shot so LUT based gamma corrections algorithms were used for contrast improvement of the images. LUT (Look up Table) was formulated suing the maximum value of intensity and the gamma values based on an input image. The actual values of brightness of an image I was mapped to I by using the formulated LUT. A procedure was suggested by the authors to set automatically the gamma values depending on the features of the actual image. The results showed that in low and high contrast the value for gamma was selected effectively. 2. Removal of the background, only foreground objects were kept while noise was removed to get the more accurate results. The actual image was blurred by adding the Gaussian kernel 
for removal of noise. The image was converted to CIELab color space that was very effective to identify objects depends on salient properties. To adjust the overall object, boundary properties were mixed with the threshold image by the help of canny edge detector. The labeling method was used to pick the biggest object among all different objects from an output image. Algorithms for image dilation and erosion were used to adjust the objects by the help of disk element of fixed size. To cover the inner holes the resultant image was filled. The final image was mask of the whole object to in an image that was used as the threshold for actual image segmentation. The resultant image was now free from the background. 3. Detecting damaged area with approximation of its severity, detection of damage was done only.

In [8], the authors have been formed an automatic system to classify brown spot and the blast diseases of the leaves of the rice crop depending upon the morphological changes happened to the plants by the diseases. SVM and Bayes' classifiers are used for the classification purpose. 1000 test spot images of the damaged rice leaves are picked. Results showed 79.5\% accuracy for Bayes' and $68.1 \%$ accuracy for SVM classifiers. The authors proposed a system which is consisted of the following steps: 1. Acquisition of the image. The images are taken by the digital camera first. There are samples of normal leaves and the affected leaves (brown spot, blast). 2. Preprocessing. First the captured images were classified as affected and unaffected leaves. Distribution of color was the same for unaffected leaves, but for the affected leaves the distribution of color was not uniform. This is because the values of the pixels of the affected leaves were totally different form the pixel values from the normal leaves. The image quality was improved by applying the mean filter. 3. Segmentation of the image. Otsu's thresholding algorithm was used for the segmentation [9]. To select threshold values Hue plane image was used. Segmentation was done and color values according to segmented regions were taken. 3. Selection and Extraction of the features. The morphological changes happened due to different diseases were observed. It was noticed that the shape was oval for the brown spots and blast spots were elliptical in shape, and these shapes were distorted according to the severity of the diseases. For center and edges different colors of spots were there. Radial distribution from center to the edges was taken as the distinguishing feature of the spot. Hue represented true colors, while hue distribution values were stored in the vectors. 4. Classification. Two types of classifications were done. One is the classification between the unaffected and the affected leaves and another was the classification between the affected leaves. During the classification between the affected and the unaffected leaves probability of occurrences of different gray levels was represented by the histogram in an image. A peak in the histogram means the high probability of a specific gray level. The probability of a specific gray level is very high for unaffected leaves because the gray level is almost same for all the pixels. But an affected image will have a high probability for at least two different gray levels, one for the affected portion and another is for unaffected portion. So the histogram for an image with single peak means unaffected image and the histogram for an image with two peaks means affected image. For classification between the affected leaves, classifiers depend upon the Bayes' theorem and SVM were used for classification and differences between the affected leaves. Based on the likelihood of a specific pattern within that class, the belongingness of a specific pattern was measured in a specific class by the Bayes' classifier [10]. 10 different combinations of training and testing sets were applied achieving $79.5 \%$ accuracy. Now classification by SVM with 10 fold cross validation was done for the same data set getting $68.1 \%$ accuracy for classification.

In [11], the authors determined the damage ratio of cotton leaf caused by the pests. Image enhancement and image filtering algorithms for cotton pests detection was studied in the given paper. For enhancement of contrast of an image and edges of the background of an image, histogram equalization was used in this paper, because the edges of the background image were darker. In resultant image there was noise pollution that was added to an image because of some factors like temperature of sensors and intensity of light that made difficult the process of segmentation. Filtering could be done by the spatial filtering and frequency domain filtering. Different filters were used for different images but results of spatial linear filters were proved good for leaf cotton images diseased by pests. By taking care about the important features of the image, space non linear filters were able to get the good results and it's convenient also. Frequency domain filters were able to get the better visual effects but they were not giving good results for machine processing. For color images, color filters were also used for good visual effects and machine processing affects. Comparisons of different color models like RGB, HSI and $\mathrm{YCbCr}$ were done by the authors. During comparisons it's proved that $\mathrm{YCbCr}$ is a good color model for feature extraction. The damage ratio selected was reliable. For future, more algorithms can be developed for more accuracy for determining the disease degree.

In [12], the authors proposed a procedure for measurement of severity degree of brown spot in cassava leaves. Samples of cassava leaves were taken from Kamphaengsaen Campus. 6 months old plants samples were taken. The assessment of brown leaf disease was done by the area diagrams. All leaves were assessed with the help of seven rater visually. Image analysis methodologies were used based on discretion of the individual raters for assessing independently. Severity assessment was done in three steps: 1 . capturing of image. Digital camera (IXY55 model) with under illuminated controller box and joined to computer by USB port was used to capture 48 image samples with 1600 pixels horizontally and 1200 pixels vertically. RGB color images with JPEG format were captured. 2. Enhancement of image. For analysis of images image processing 
toolbox was used for MATLAB. The original images were resized by 640 pixels horizontally and 480 pixels vertically. Then RGB images were transformed into HSI format. HSI color space was tolerant for variations of intensities. 3. Segmentation of image and extraction of features. The intensity (I) of the image was segmented by Otsu's method, which was based on the histogram of the tonal image. Form pixel combinations the leaf area was extracted and calculated as total leaf area (AL). The number of brown spots and disease area (AD) was calculated by segmentation oh Hue $(\mathrm{H})$ in the histogram. Now noise was spread all over the image and brown spots were extracted. Noise removal was done by erosion and dilation processes. To measure the number of spots and find out the diseased area, the components were labeled by the image in 2-D binary image (8- connected objects methodology). In the end, percentage of infection (PI) was measured by the formula: $\mathrm{PI}=(\mathrm{AD} / \mathrm{AL}) * 100$. Based on different values of Hue, the affected images were extracted from the healthy area of the Hue image. For better segmentation a proper threshold value is needed. Based on the guidelines of segmentation of the affected pixels from the healthy part, the threshold value was chosen manually. When segmentation of healthy and infected portions was done then PI was calculated. For assessing the brow spots 4 images of area diagram key were tested and PI value was calculated. Analysis of area diagram key was done by the processing of image which showed that number and position of the spots were correct but percentage of infection area was different specifically in the severity levels 1-3. The achieved values from the image analysis were used as new criteria for further severity classification. Assessment of brown leaf spot (BLS) was done by comparing different assessment procedures. The percentage difference between the affected cassava leaves by the digital image analysis and area diagram key was determined. PI value was less than $4 \%$, except on sample with $8.20 \%$ when classification of 48 samples was done by digital image analysis. At level 1, classification of BLS was same for both the methods.

In [13], authors developed a digital image processing technique by online system development in java applets that is time efficient as compared to other techniques like visual inspection, microscopic and biochemical methodologies. In color image processing red, green and blue colors were specified. RGB cannot perceive the color differences as humans do so in image processing RGB is transformed in other color spaces which are similar to human visual system [14] and [15]. For this purpose HIS (Hue), (Saturation) and Luminance (Intensity) was used. In this paper a method was suggested for color image segmentation using perceptual spaces and prewitt algorithm for segmentation with a computational system of Java Applets to perform image processing through internet to identify and prevent the diseases in chili peppers. Lightness or darkness of an image is actually the Luminance and is expressed as \% age from $0 \%$ (black) to white (100\%). The color transmitted through an object or color reflected from is the Hue, and was measured as polar (angle in degrees between 0 degree and 360 degrees. Hue is defined by the name of the color like, orange, red, green, etc. Saturation (chroma) is the strength or purity ofa color and it shows the amount of white in proportion to the pitch and is measured as \% age between $0 \%$ (gray) to $100 \%$ ( fully saturated). Saturation increases as the edges arrived. Chromaticity is defined by the coordinates of Hue and Saturation. Luminance and chromaticity characterized a color. A bitmap image (bmp) of 24 bits was used here. Web application system was used in java applets. For image segmentation threshold and perwitt algorithm was used. Perceptual space, Hue, Luminance and Saturation was used. In segmentation method first RGB conversion was done in to HSL (Hue, Saturation and lightness), HIS (Hue, Saturation and Intensity) and HSV (Hue, Saturation and Value). For noise removal preprocessing was performed by the opening or lock reconstruction. Color segmentation procedure was applied with each perceptual space and for each channel i.e, Hue, Luminance and Saturation. Then threshold was applied to the image. Then perwitt filter was applied to the image. Conversion from perceptual space to RGB was done. The results of the colored segmentation achieved from the computer system using java applets on real time or remote internet were then analyzed. The details of the diseases were highlighted by the system. Comparisons for all perceptual spaces were done to identify and prevent the chili peppers diseases. Form the results achieved the plant pathology expert could use any method for disease identification.

In [16], the authors suggested a grading system for the disease of leaves of the plants. Leaves of Pomegranate were taken for experiments. The proposed system was divided in to five steps 1. Acquisition of the image: It is the process of digitization and storage of an image. All the captured images were stored in JPEG format. Authors have visited several pomegranate farms in India to capture the images. 2. Preprocessing of the image: Image processing is the type of signal processing having image as input e.g, photo or video frame and image or set of characteristics of an image as an output. Image resizing and filtering were the phases of preprocessing. The acquired images were resized first to minimize the storage and computational burden for later preprocessing. Noise removal or reduction was performed for better segmentation and feature extraction of the diseased areas. This was done by the use of Gaussian filter to get the suitable image. 3. Colored image segmentation: It the process of partitioning the digital image in to separated portions so that these are more meaningful for analysis. In the given paper k-means clustering method was used for image segmentation is performed in 5 phases.: I. image reading. II. Image conversion from RGB color space to $\mathrm{L}^{*} \mathrm{a} \mathrm{b}^{*}$ color space. The extent of disease was determined from PI through Fuzzy logic. By this transformation the visual difference in the RGB was quantified. III. Classification of colors in to $\mathrm{a}^{*} \mathrm{~b}^{*}$ by Kmeans clustering. Each object have a location in space in K-means. The interested elements were the pixels with the values $\mathrm{a}^{*} \mathrm{~b}^{*}$, so $* \mathrm{~b}^{*}$ contained all the color 
information. The set of (cluster-index and cluster center) was the result of the k-means clustering. IV. Labeling each pixel of an image by the results obtained from kmeans. K-means returned index relating to cluster for each element and labeled every pixel with its cluster index. V. Creation of the image for segmentation of the original image by the color. Segmentation was done by extracting one of the clusters consisted of diseased area. The result was saved and picked for AD. 4. Calculation of AT and AD. Transformation of the original resized image was done to $\mathrm{ON}$ the pixels related to the image of leaf. Now total leaf area(AT) was calculated from this image. From color segmentation, the resultant image having affected area was used to calculate AD (total diseased area). 5. Grading of disease by fuzzy logic. When $\mathrm{AT}$ and $\mathrm{AD}$ was calculated then PI(percentage infection $)$ could be calculated by : $\mathrm{PI}=(\mathrm{AD} / \mathrm{AT}) * 100$.

In [17] authors done research on cotton mite diseases by using thresholding and color features methods. The proposed method is divided in to three steps, 1. Extraction of the spots and stems from the green plants. 2. Detection of the special features in gray histogram and conversion in to 8-bit gray scale image by a single thresholding of the segmented images. 3. Comparing the areas with spots to the stems and segmentation of binary images with the help of area thresholding. According to authors cotton is very economic and important crop in china that plays a vital role in agricultural productions. So to increase the cotton production is the big issue, that's why the authors selected the cotton for their research. All the cotton mite disease images were captured in June and July 2011 by the digital camera. All those images were converted in to JPEG format and saved in the computer. The algorithm was written in $\mathrm{C}++$, Visual $\mathrm{C}++6.0$. With the complicated background, differentiation was done between the spotted area and non spotted area depending upon Excess green features 2G-R-B. Then conversion of segmented images was done in to 8-bit gray scale images by single threshold depending upon special features depicted by the gray histogram. Finally comparison of the disease spot portions with the stem was performed to do segmentation of the disease spots with analogous disease spot parts by the area threshold. Segmentation process had three stages: a. Segmentation of analogous disease spot part with the non disease spotted parts having complicated backgrounds. The images were divided in to two parts first, one is the green area (including normal leaves and weeds), and another is non green area (the analogous disease spotted part consisted of mite disease spotted parts and the stems). The stems and mite disease spotted portions resembled in color therefore to segment the healthy stem areas and the mite disease areas were a difficult task. That's why first analogous mite disease portion was segmented though the color features from the original image. Then the disease spotted portions from the analogous mite portion were extracted with the help of area threshold technique. In cotton mite diseases other color spaces were giving incorrect results so, Excess green feature $2 \mathrm{G}-\mathrm{R}-\mathrm{B}$ was used to extract the disease spotted part from the original image. B. Binarization of analogous disease spotted parts and the non disease spotted parts. When processing was done, different gray scale images were achieved. Histograms depicted that two peaks in two different gray scale portions were for the two parts (disease spotted pats with values 255 and non disease spotted parts with grayscale values less than 120). Now by these features the targeted parts could be extracted from the background by applying thresholding. Black pixels represented the analogous disease spotted part having mite disease spots and stems and white pixels represented non disease spotted areas. Experimental results showed that single thresholding segmented the analogous diseases spotted parts effectively. C. Segment the mite disease parts and the stems: form the experiments the authors found that the leaves were generally affected by the diseases and the stems were acting as independent items in case of cotton mite diseases. When further amplifications were done on binary and segmented regions, it was found that the segmented images were made up of big and independent 8 - connected areas. It was found that connected areas of stems were bigger than that of disease spotted areas. Depending upon the differences between the two areas though number of pixels, the authors chosen the correct value for threshold to remove stem areas by the help of area thrersholding and the areas that were remaining were the disease spotted areas. Experimental results showed that for 30 color images of cotton mite diseases the error extraction rate was $52.1 \%$ and the right extraction rate could reach $94.79 \%$.

In [18] the authors used image color transformation, image smoothening and diseases spot segmentation to detect diseases in plant's leaves. The phases are: i. image color transformation: The intensity of leaf vein and the color of spot of disease were different in leaves. To reduce the effect of veins, different color space models like, RGB, YCbCr, HSI \& CIELAB were used. II. Image Smoothing: Median filter was used to remove the noise. III. Segmentation of spotted diseases. Selection of threshold of the gray level is required to extract the spotted disease from the leaves. For (experiments with different color disease spots), black, brown, white and gray colored spots were identified correctly by using CIELAB. For future, same color of disease spot and vein can be taken because this work is not done here.

In [19], the authors have done the classification for three different classes (Cercospora beticola, Uromyces betae and healthy leaf portions. Above $90 \%$ classification rate was achieved for all the three classes. For experiments 30 sugar beet plants were selected having 4 developed leaves. 15 plants with the rust fungus Uromyces betae and15 plants having leaf spot pathogen Cercospora beticola were selected. Both types were limited to the leaves and were fungal infections. Two leaves were taken from the leaves and marked for observing the disease development. Images were acquired in a controlled illumination conditions within a lab. The experiments were done on single leaves. For three weeks each day pictures of sugar beet were taken. Then images from multispectral camera and RGB were mixed for 
having a $3 \mathrm{D}$ model of the leaves. Different values were achieved: (i) From 4 RGB images, $4 * 9$ blue (ii) from the one multispectral image and 4 RGB images, $5 * 9$ green (iii) $5 * 9$ red same as with green and (iv) From the multispectral image, $1 * 9$ infrared. 15 different values were taken from these having 1 blue, 1 green and 1 red, from one of the RGB images and the infrared channel from the MS camera as the features for classification. Green and Red data from MS camera was removed as they were not adding any enhancement in the classification results. Test data set included pictures after inoculation of (10-19) days. 55 rectified images from the Cercospora dataset and 73 from the Uromyces data sets were observed. These pictures were divided in to $64 * 64$ pixels's patches which showed that only 4 conditions were possible: (i) white background, (ii) leaf area background, (iii) healthy leaf portion with no background and (iv) healthy and infected leaf portions. It was easy to determine the difference between the white background and green leaves [20]. For classification three different techniques were used: (i) 1.2 pixel based approach, when classes of pixels were found pixel by pixel and assigned independently of each other, for that $(\mathrm{kNN}) \mathrm{k}$ - nearest neighbor technique was used due to data distribution independency, simplicity and adaptive Bayes classification by Gaussian mixture models. $\mathrm{kNN}$ classifier could determine the bounds of Bayes errors to separate the different classes. The data could be defined by multiple Gaussian distributions in Adaptive Bayes classification using Gaussian mixture models (GMM), The better classification results were obtained with these number Gaussian distributions per class: (a) healthy leaf area: 2 distributions, (b)Cercospora beticola: 3 distributions and (c)Uromyces betae: 1 distribution. The $\mathrm{P}$ (l) priori probability was measured in the classifier training stage. $\mathrm{P}(\mathrm{l})$, was calculated for each image at the classification level by pre classifying the image. (ii) Global method when pixel classes were found and assigned at the same time, where CRF (Conditional Random Field) method was used. The results obtained by GMM were better than expected and as compared to kNN. The results achieved by the GMM for Cercospora beticola and the healthy leaf area class were according to the hypothesized $90 \%$. The results obtained by CRF classification were more homogeneous and smooth than expected and as compared to GMM.

References [21-26] reviewed different techniques in different domains and reported their critical evaluations along with a workable framework where necessary.

\section{CRITICAL REVIEW}

\begin{tabular}{|c|c|c|c|c|}
\hline Ref. & Technique Used & Focus Area & Strengths & Weaknesses \\
\hline$[1]$ & $\begin{array}{l}\text { Otsu's method, } \mathrm{K}-\text { means } \\
\text { clustering, ANN, Color } \\
\text { Occurrence method }\end{array}$ & $\begin{array}{l}\text { Accurate \& Fast plant Diseases } \\
\text { Detection and Classification }\end{array}$ & $\begin{array}{l}\text { 1. Color Occurrence method } \\
\text { provides extra features than the } \\
\text { gray scale method. } \\
\text { 2.Accuracy is improved by ANN. }\end{array}$ & $\begin{array}{l}\text { 1. K- values prediction is } \\
\text { difficult. .K-means is not good for } \\
\text { global clustering. } \\
\text { 2. ANN must be trained, data } \\
\text { can't be added later. }\end{array}$ \\
\hline [2] & $\begin{array}{l}\text { Vector median filter. Open } \\
\text { and close operations }\end{array}$ & $\begin{array}{l}\text { Grading methods of crop } \\
\text { diseases based on image } \\
\text { processing }\end{array}$ & $\begin{array}{l}\text { 1. Good edges were got by vector } \\
\text { median filter. } \\
2 \text { Open, close operations } \\
\text { removed the noise }\end{array}$ & $\begin{array}{l}\text { 1. The median operation is } \\
\text { applied to each pixel without } \\
\text { taking care that this is damaged or } \\
\text { not in median filter. }\end{array}$ \\
\hline [3] & $\begin{array}{l}\text { SVM (Support Vector } \\
\text { Machine, Median Filter. }\end{array}$ & $\begin{array}{l}\text { Detection and classification of } \\
\text { pests in green house using } \\
\text { image processing }\end{array}$ & $\begin{array}{l}\text { 1. The flaws in static images and } \\
\text { sticky traps are covered. }\end{array}$ & $\begin{array}{l}\text { 1. The median operation is } \\
\text { applied to all the pixels. }\end{array}$ \\
\hline [4] & HSI, SGDM & $\begin{array}{l}\text { Detection, classification of } \\
\text { plant leaf diseases }\end{array}$ & $\begin{array}{l}\text { 1. HSI is a human perception } \\
\text { model. }\end{array}$ & $\begin{array}{l}\text { 1. HSI not supported standards } \\
\text { like JPEG. }\end{array}$ \\
\hline [5] & $\begin{array}{l}\text { K-means } \\
\text { Algorithm }\end{array}$ & $\begin{array}{l}\text { Automatic plant pest detection } \\
\text { and recognition using k-Means } \\
\text { clustering algorithm and } \\
\text { correspondence filters }\end{array}$ & $\begin{array}{l}\text { 1. Good accurate pest's detection } \\
\text { and recognition in different } \\
\text { shapes, sizes, orientation and } \\
\text { positions. }\end{array}$ & $\begin{array}{l}\text { 1. For different sizes of clusters } \\
\mathrm{K} \text {-means is not good. To predict } \\
\text { values is a problem. }\end{array}$ \\
\hline [6] & $\begin{array}{l}\text { Nearest } \\
\text { classifiers }\end{array}$ & $\begin{array}{l}\text { Statistical Methods for } \\
\text { Quantitatively } \\
\text { Fungal Disease from Fruits' } \\
\text { Images }\end{array}$ & $\begin{array}{l}\text { 1. Nearest neighbor classifiers } \\
\text { were very simple } \\
\text { 2. Block wise feature improved } \\
\text { the accuracy rate. }\end{array}$ & $\begin{array}{l}\text { 1.Only identified the fungal } \\
\text { diseases and only for the fruits }\end{array}$ \\
\hline [7] & $\begin{array}{ll}\text { LUT depending } & \text { gamma, } \\
\text { Fuzzy } & \text { c-means } \\
\text { segmentation } & \\
\end{array}$ & $\begin{array}{l}\text { Automatic estimation of live } \\
\text { coffee leaf infection based on } \\
\text { DIP }\end{array}$ & $\begin{array}{l}\text { 1. It not only identified the } \\
\text { destroyed part of the leaf, but also } \\
\text { improved the color contrast. }\end{array}$ & $\begin{array}{l}\text { 1. It only works for the leaves and } \\
\text { the coffee leaves. }\end{array}$ \\
\hline [8] & $\begin{array}{l}\text { SVM and } \quad \text { Bayes' } \\
\text { classifiers, } \\
\text { thresholding algorithm }\end{array}$ & $\begin{array}{llr}\text { Classification } & \text { of Rice } & \text { Leaf } \\
\text { Diseases } & \text { Based } & \text { on } \\
\text { Morphological Changes } & \end{array}$ & $\begin{array}{l}\text { 1.More time efficient as compared } \\
\text { to SVM, because both classifiers } \\
\text { i.e Bayes' and SVM was used }\end{array}$ & $\begin{array}{l}\text { 1. Misclassification may be } \\
\text { happened because of shadow } \\
\text { effect or color distortion of aging } \\
\text { leaves. }\end{array}$ \\
\hline [11] & $\begin{array}{l}\text { HSI ,RGB and } \mathrm{YCbCr} \\
\text { color models }\end{array}$ & $\begin{array}{l}\text { Cotton Pests and Diseases } \\
\text { Detection based on Image } \\
\text { Processing }\end{array}$ & $\begin{array}{l}\text { 1. YCbCr is a good color model } \\
\text { for feature extraction. 2.The } \\
\text { damage ratio selected was reliable }\end{array}$ & $\begin{array}{l}\text { 1. Indoor experiments were done } \\
\text { only but in real life noise } \\
\text { interference and cotton shadow } \\
\text { are the hurdles inaccurate } \\
\text { measurement of damage degree. }\end{array}$ \\
\hline [12] & Otsu's method & $\begin{array}{l}\text { Assessment of the Severity of } \\
\text { Brown Leaf Spot Disease in } \\
\text { Cassava using Image Analysis }\end{array}$ & $\begin{array}{l}\text { 1. DIP will eliminate all the draw } \\
\text { backs like errors and more time } \\
\text { consumption of manual ways. }\end{array}$ & $\begin{array}{l}\text { 1. Infection degree measured by } \\
\text { digital image analysis for other } \\
\text { levels was less than the area } \\
\text { diagram key. }\end{array}$ \\
\hline
\end{tabular}




\begin{tabular}{|c|c|c|c|c|}
\hline [13] & Perwitt filter & $\begin{array}{l}\text { Color image segmentation } \\
\text { using perceptual spaces } \\
\text { through applets for determining } \\
\text { and preventing diseases in chili } \\
\text { peppers }\end{array}$ & $\begin{array}{l}\text { 1. Draw backs like expensive } \\
\text { equipments, training, personnel } \\
\text { and more time by other methods } \\
\text { like naked eye inspection, } \\
\text { microscopic and biochemical } \\
\text { were overcome by the given } \\
\text { method. } \\
\text { 2. perwitt gave the better results } \\
\text { than Sobel and Laplacian }\end{array}$ & $\begin{array}{l}\text { 1. HSL and HSV could not } \\
\text { identify the diseased area but } \\
\text { identified only the contour of the } \\
\text { leaves, 2.HSV only identified } \\
\text { those areas that do not belong to } \\
\text { the infected parts as compared to } \\
\text { HSI. } \\
\text { 3. Perceptual spaces were not so } \\
\text { good because each perceptual has } \\
\text { to be selected. }\end{array}$ \\
\hline [16] & $\begin{array}{l}\text { K-means } \\
\text { Algorithm }\end{array}$ & $\begin{array}{l}\text { Grading of Leaf Diseases } \\
\text { through Fuzzy Logic \& } \\
\text { Machine Vision }\end{array}$ & $\begin{array}{l}\text { 1. Fuzzy logic is effective in } \\
\text { applications like texture analysis. }\end{array}$ & $\begin{array}{l}\text { 1. User has to choose the exact } \\
\text { value of } k \text { that is difficult. }\end{array}$ \\
\hline [17] & Area thresholding, & $\begin{array}{l}\text { Image segmentation method } \\
\text { for cotton mite disease based } \\
\text { on color features and area } \\
\text { threshholding. }\end{array}$ & $\begin{array}{l}\text { 1. Area thresholding is real time } \\
\text { and robust. }\end{array}$ & $\begin{array}{l}\text { 1. Only restricted for cotton mite } \\
\text { diseases. } \\
\text { 2. Chances of wrong extraction. }\end{array}$ \\
\hline [18] & $\begin{array}{l}\text { HSI, RGB, YCbCr and } \\
\text { CIELAB color models. }\end{array}$ & $\begin{array}{l}\text { Disease Spot Detection in } \\
\text { plants by Color Transform } \\
\text { Approach. }\end{array}$ & $\begin{array}{l}\text { 1. For (experiments with vein } \\
\text { disturbance), by threshold on A of } \\
\text { CIELAB, all diseases of spots } \\
\text { were identified. }\end{array}$ & $\begin{array}{l}\text { 1. Disease spots could not } \\
\text { identified accurately by applying } \\
\text { threshold on RGB } 2 \text {. Results was } \\
\text { depending on background type. }\end{array}$ \\
\hline [19] & $\begin{array}{l}\text { CRF(Conditional Random } \\
\text { Field), Gaussian mixture } \\
\text { models (GMM)and kNN } \\
\text { classifier }\end{array}$ & $\begin{array}{l}\text { Automatic methods } \\
\text { classification to identify leaf } \\
\text { diseases from multispectral } \\
\text { images, }\end{array}$ & $\begin{array}{l}\text { 1. The results obtained by GMM } \\
\text { were better than kNN. } \\
\text { 2. CRF removed the draw backs } \\
\text { of pixel wise classification. }\end{array}$ & $\begin{array}{l}\text { The flaws of a pixel wise } \\
\text { classifier, the isolated pixels were } \\
\text { misclassified and neighboring } \\
\text { pixels were allocated to different } \\
\text { classes, even when they belonged } \\
\text { to the same class. }\end{array}$ \\
\hline
\end{tabular}

\section{CONCLUSION AND FUTURE WORK}

In this paper we have presented literature review of automatic and rapid plant's disease segmentation techniques and algorithms. The purpose was to present the existing techniques of plant's disease segmentation. Time and accuracy are the two important things to be considered. Accuracy can be improved by the use of different methods using image processing techniques, as compared to manual systems. Time is also saved by these new techniques.

Among all these different techniques best techniques will be analyzed who have the maximum benefits. But in spite of the maximum benefits every technique has certain limitations. So to overcome the drawback of different techniques fusion of different techniques is the good idea. In the future we will fuse two or three techniques to get accurate results with fastest speed.

\section{REFERENCES}

[1] H.Al-Hiary, S. Bani-Ahmad, M.Reyalat, M.Braik \& Z.AlRahamneh, "Fast and Accurate Detection and Classification of Plant Diseases", International Journal of Computer Applications, Vol. 17, No.1, pp. 31-38.March 2011.

[2] Y. Tian, L. Wang and Q. Zhou, "Grading method of Crop disease based on Image Processing", Computer and computing technologies in agriculture 427-433, 2011.

[3] R.G. Mundada, Dr. V.V. Gohokar, "Detection and classification of Pests in Green House using Image Processing", IOSR Journal of Electronics and Communication Engineering (IOSR-JECE) Vol. 5, Issue 6, PP 57-63, 2013.

[4] S. Ananthi \& S.V. Varthini, "Detection and Classification of Plant Leaf Diseases", International Journal of Research in Engineering \& Applied Sciences, Vol. 2, Issue 2, pp.763-773, February 2012.
[5] F. Fina, P. Birch, R. Young, J. Obu, B. Faithpraise and C. Chatwin, "Automatic Plant Pest detection and recognition using k-means clustering algorithm and correspondence filters", International Journal of Advanced Biotechnology and Research, Vol. 4, Issue 2, pp 189-199, 2013.

[6] J. D.Pujari, R. Yakkundimath \& A.S.Byadgi, "Statistical Methods for Quantitatively Detecting Fungal Disease from Fruits' Images", International Journal of Intelligent Systems \& Applications in Engineering Advanced Technology \& Science, vol.1 no.4, pp.60-67, 12th Dec2013.

[7] E. Hitimana \& O. Gwun, "Automatic estimation of live Coffee leaf infection based on Image processing techniques", Second International Conference on Signal, Image Processing and Pattern Recognition, Sydney, Australia, pp. 255-266, 2014.

[8] S. Phadikar, J. Sil, and A. K. Das, "Classification of Rice Leaf Diseases Based on Morphological Changes", International Journal of Information and Electronics Engineering, Vol. 2, No. 3, pp. 460-463, May 2012.

[9] J. N. Kapur, P. K. Sahoo \& A.K.C. Wong, "A New Method for Gray Level Picture Threshold Using the Entropy of the Histogram," Graphical Models and Image Processing, 29, pp. 273-285. 1985.

[10] T. Touanf and R. C. Gonzalez, "In: Pattern Recognition Principles. London," UK, Addition Wesley Publishing Company. pp. 110-1543, 1974.

[11] Q. He, B. Ma, D. Qu, Q. Zhang, X. Hou, J. Zhao, "Cotton Pests and Diseases Detection based on Image Processing", TELKOMNIKA, Indonesian Journal of electrical engineering, Vol. 11, No. 6, pp. 3445 3450, June 2013.

[12] W. Abudullakasim \& J. Unartngam, "Assessment of the Severity of Brown Leaf Spot Disease in Cassava using Image Analysis", The International conference of the Thai Society of Agricultural Engineering, 2012.

[13] Gudiño, J. Gudiño-Bazaldúa, J. L. Rojas-Rentería, V. Rodríguez-Hernández and V.M. Castaño, "Color image segmentation using perceptual spaces through applets for determining and preventing diseases in chili peppers", African Journal of Biotechnology Vol. 12,no.7, pp. 679688, 2013. 
[14] F. Ortiz, F. Torres, E. Juan \& N. Cuenca, "Colour mathematical morphology for neural image analysis", Real-Time Imaging 8, pp. 455-465, (2002).

[15] J.Yang, C. Liu \& L.Zhang, "Polar space normalization: Enhancing the discriminating power of polar spaces for face recognition", Pattern Recognit. 43, pp. 1454-1466, (2010).

[16] S. S. Sannakki, V. S. Rajpurohit, V. B. Nargund, A.R. Kumar \& P. S. Yallur, " Leaf Disease Grading by Machine Vision and Fuzzy Logic", International Journal Computer Technology Applications, vol.2 ,no.5, pp. 17091716. 2011.

[17] D. Zhihua, W. Huan, S. Yinmao \& W. Yunpeng," Image segmentation method for cotton mite disease based on color features and area thresholding", Journal of Theoretical and Applied Information Technology, Vol. 48, No.1. 2013.

[18] P. Chaudhary, A. K. Chaudhari, Dr. A. N. Cheeran \& S. Godara, "Color Transform Based Approach for Disease Spot Detection on Plant Leaf", International Journal of Computer Science and Telecommunications, Vol. 3, Issue 6, June 2012.

[19] S. D. Bauer , F. Korc, W. Fo"rstner, "the potential of automatic methods of classification to identify leaf diseases from multispectral images", Precision Agriculture, vol. 12, No.3, pp.361-377, 26 January 2011

[20] S. D. Bauer, F. Korc, W. Fo"rstner, "Investigation into the classification of diseases of sugar beet leaves using multispectral image", In E. J. van Henten, D. Goense, \& C. Lokhorst (Eds.), Precision agriculture '09 (pp. 229238). Wageningen: Wageningen Academic Press, 2009.

[21] Khan, MNA., Khalid M., ulHaq S., Review of Requirements Management Issues in Software Development. International Journal of Modern Education \& Computer Science, 5(1), (2013).

[22] Abbasi, A. A., Khan, M. N. A., \& Khan, S. A. (2013). A Critical Survey of Iris Based Recognition Systems. Middle-East Journal of Scientific Research, 15(5), 663668.

[23] Zia, A., \& Khan, M. N. A. (2012). Identifying key challenges in performance issues in cloud computing. International Journal of Modern Education and Computer Science (IJMECS), 4(10), 59.

[24] Ul Haq, S., Raza, M., Zia, A., \& Khan, M. N. A. (2011). Issues in global software development: A critical review. An Appraisal of Off-line Signature Verification
Techniques 75 Copyright (C) 2015 MECS I.J. Modern Education and Computer Science, 2015, 4, 67-75 Journal of Software Engineering and Applications, 4(10), 590.

[25] Zia, A., \& Khan, M. N. A. (2013). A Scheme to Reduce Response Time in Cloud Computing Environment. International Journal of Modern Education and Computer Science (IJMECS), 5(6), 56.

[26] Masood, M. A., \& Khan, M. N. A. (2015). Clustering Techniques in Bioinformatics. I.J. Modern Education and Computer Science, 2015, 1, 38-46.

\section{Authors' Profiles}

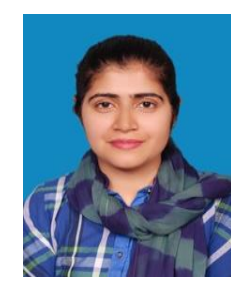

Rabia Masood is s lecturer at National University of Modern Languages and Sciences, Islamabad, Pakistan since 2007. She obtained MS degree in Computer Science from Shaheed Zulfikar Ali Bhutto Institute of Science and Technology, Islamabad, Pakistan. Her research areas include artificial intelligence, digital image processing and data mining techniques.

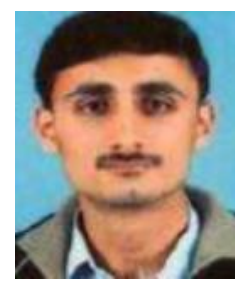

S. A. Khan received his BS computer science degree from the PMAS University of Arid Agriculture, Pakistan. He completed his MS degree in computer science from SZABIST University Islamabad, Pakistan. His research areas are pattern recognition, face analysis and digital image processing.

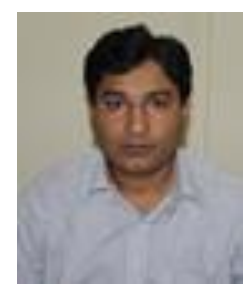

M.N.A. Khan obtained D.Phil. degree from the University of Sussex, Brighton. His research interests are in the fields of software engineering, cyber administration, digital forensic analysis and machine learning techniques.

How to cite this paper: Rabia Masood, S.A. Khan, M.N.A. Khan,"Plants Disease Segmentation using Image Processing", International Journal of Modern Education and Computer Science(IJMECS), Vol.8, No.1, pp.24-32, 2016.DOI: 10.5815/ijmecs.2016.01.04 